\title{
1 Sonogenetic modulation of cellular activities using an engineered auditory-sensing
}

2

3 Yao-Shen Huang ${ }^{1 *}$, Ching-Hsiang Fan $^{2 *}$, Ning Hsu ${ }^{1 *}$, Chun-Yao Wu ${ }^{2}$, Chu-Yuan Chang ${ }^{1}$, Shi-

4 Rong Hong ${ }^{1}$, Ya-Chu Chang ${ }^{1}$, Anthony Yan-Tang $\mathrm{Wu}^{3,4,5}$, Vanessa Guo ${ }^{3}$, Yueh-Chen Chiang ${ }^{1}$,

5 Wei-Chia Hsu', Nai-Hua Chiu ${ }^{2}$, Linyi Chen ${ }^{1,6}$, Charles Pin-Kuang Lai ${ }^{3,4,7}$, Chih-Kuang Yeh²,

6 Yu-Chun Lin ${ }^{1,6}$

7

$8{ }^{1}$ Institute of Molecular Medicine, National Tsing Hua University, Hsinchu, 300, Taiwan

$9 \quad{ }^{2}$ Department of Biomedical Engineering and Environmental Sciences, National Tsing Hua

10 University, Hsinchu, 300, Taiwan

11 3'Institute of Atomic and Molecular Sciences, Academia Sinica, Taipei, 106, Taiwan

12

13

14

15

$17{ }^{7}$ Genome and Systems Biology Degree Program, National Taiwan University and Academia

Sinica, Taipei, 106, Taiwan.

19

$20 *$ These authors contributed equally to this work

21 Correspondence and requests for materials should be addressed to ckyeh@mx.nthu.edu.tw

22 and ycl@life.nthu.edu.tw 


\section{Abstract}

25 Biomolecules that respond to different external stimuli enable the remote control of genetically modified cells. Chemogenetics and optogenetics, two tools that can control cellular activities via synthetic chemicals or photons, respectively, have been widely used to elucidate underlying physiological processes. These methods are, however, very invasive, have poor penetrability, or low spatiotemporal precision, attributes that hinder their use in therapeutic applications. We report herein a sonogenetic approach that can manipulate target cell activities by focused ultrasound stimulation. This system requires an ultrasound-responsive protein derived from an engineered auditory-sensing protein prestin. Heterogeneous expression of mouse prestin containing two parallel amino acid substitutions, N7T and N308S, that frequently exist in prestins from echolocating species endowed transfected mammalian cells with the ability to sense ultrasound. An ultrasound pulse of low frequency and low pressure efficiently evoked cellular calcium responses after transfecting with prestin(N7T, N308S). Moreover, pulsed ultrasound can also non-invasively stimulate target neurons expressing prestin(N7T, N308S) in deep regions of mice brains. Our study delineates how an engineered auditory-sensing protein can cause mammalian cells to sense ultrasound stimulation. Moreover, owing to the great penetration of low-frequency ultrasound ( $400 \mathrm{~mm}$ in depth), our sonogenetic tools will serve as new strategies for non-invasive therapy in deep tissues of large animals like primates. 
Approaches that can non-invasively stimulate target cells buried in the deep tissues are including photons, chemicals, radio waves, and magnetic fields have been used to stimulate target cells implanted with stimulus-responsive proteins or nanoparticles ${ }^{1-4}$. However, these strategies suffer from several drawbacks including invasiveness, poor spatiotemporal precision, or low penetration depth, which greatly hinder their potential use in clinical therapy. To overcome these long-standing problems, we aim to use focused ultrasound (FUS) as a stimulus to remotely control cellular activities because it can non-invasively deliver acoustic energy to deep tissues while retaining spatiotemporal coherence ${ }^{5}$. by humans $(>20 \mathrm{kHz})$. Low-frequency ultrasound waves $(<3.5 \mathrm{MHz})$ are easily transmitted through tissues, including those of bones and brains ${ }^{6}$. Owing to its deep penetrability and spatiotemporal resolution (a few cubic millimetres), ultrasound-based neuromodulation has been tested on cultured neuronal cells and in brains of various model organisms ${ }^{6-11}$. As continuous ultrasound waves or pulsed ultrasound waves of high acoustic pressure are typically needed to activate neurons, neuronal cells are likely to be weakly sensitive to ultrasound stimulus $^{8,12,13}$. To overcome this, gas-filled microbubbles (MBs) that vibrate upon ultrasound excitation have been used as ultrasound amplifiers to enhance their mechanical effects on target cells $^{14,15}$. Recently, lbsen and colleagues used MBs to transduce mechanical stimulation from ultrasound waves to neuronal cells in Caenorhabditis elegans and induced behavioural output ${ }^{16}$. The pore-forming cationic mechanotransduction ion channel TRP-4 may be involved in transducing ultrasound stimulation onto MBs attached to C. elegans ${ }^{16}$. Although this study 
verified that ultrasound-mediated neuromodulation is possible, its further development faces major roadblocks, i.e.,, MBs have a short lifespan in vivo ( $<5 \mathrm{~min}$ in the blood), and it is difficult to deliver MBs to extravascular tissues ${ }^{17}$. Compared with MBs, gas-filled protein complexes, denoted as gas vesicles, are highly stable both in vitro and in vivo and efficiently oscillate in response to ultrasound excitation. Different gas vesicle variants can serve as genetically encoded ultrasound contrast reagents to track target microbes or cells by ultrasound imaging ${ }^{18,19}$. However, it is still challenging to express and assemble prokaryotic gas vesicles

77 in mammalian cells ${ }^{5}$. Recently, several groups implanted mechanosensitive ion channels, such as Mscl and Piezo1, into in vitro cell culture systems and, with their use, successfully perturbed the cellular membrane potentials of target cells using ultrasound ${ }^{20,21}$ However, the ultrasound frequencies used in those studies are too high (30 MHz and $43 \mathrm{MHz}$ ) to be applicable for in vivo use owing to their low penetrability $(<5 \mathrm{~mm})$. Therefore, to date, there has been no sonogenetic system that uses low-frequency and low-pressure ultrasound to remotely control activities of mammalian cells that have been genetically modified. or communicate. The high-frequency auditory sensitivity and selectivity in echolocating mammals have been attributed to adaptive mechanical amplification in the outer hair cells

87 (OHCs) of their cochlea ${ }^{22}$. Prestin (also known as SCL26A5) is a transmembrane protein residing in $\mathrm{OHCs}$ that drives their electromotility and seems to be involved in the ability to 89 hear ultrasound ${ }^{23-25}$. Heterologous expression of prestin endows transfected mammalian cell 90 lines with several of the physiological hallmarks of OHCs, suggesting that prestin may inherently act as an electromechanical transducer ${ }^{26}$. Evolutionary analysis also suggests that

92 prestin is involved in ultrasound sensing of echolocating mammals ${ }^{23}$. The primary sequence of 
93 prestin is largely conserved among various mammalian species, although several specific

94 amino acid substitutions that directly affect the electromotility capacity of prestin frequently

95 occur in prestins of sonar mammals but not in those of their non-sonar counterparts ${ }^{23,24}$. Thus

96 prestin probably enhances ultrasound sensitivity in mammals, although how it does so is still

97 unclear.

98

99

100

101

102

103

104

105

106

107

108

109

110

111

112

\section{Results and discussion}

Here we first examined the amino acid sequences of prestin from six non-echolocating species and eight echolocating species. Asn at positions 7 and 308 in prestins of nonecholocating species is frequently replaced with Thr and Ser, respectively, in echolocating species (Fig. 1a). To test whether these apparently evolutionarily driven amino acid substitutions are important to adaptive ultrasound sensing, two mutations N7T and/or N308S were introduced into mouse prestin (hereafter mPrestin). The constructs used for our study were wild-type prestin (mPrestinWT); mPrestin mutants containing a single substitution, mPrestin(N7T) and mPrestin(N308S); and a mutant containing two substitutions, mPrestin(N7T, N308S). Each of these constructs was tagged with the yellow fluorescent protein Venus. Each construct was co-transfected with the calcium biosensor cyan fluorescence protein (CFP)-R-GECO into the human HEK293T cell line. The calcium influx of transfected cells was used as a readout in response to the mechanical stimulation of ultrasound wave. To simultaneously excite FUS and acquire real-time cell images, an ultrasound transducer connected to a waveform generator and an amplifier was placed on top of the live-cell imaging system. This system focuses ultrasound waves to a circle with a diameter of a few millimetres over a monolayer of cultured cells (Extended Data Fig. 1). Using this ultrasound-imaging 
116

117

118

119

120

121

122

123

124

125

126

127

128

129

130

131

132

133

134

135

136

137

138

system, we stimulated cells co-expressing CFP-R-GECO and Venus-mPrestin(N7T, N308S) or co-expressing CFP-RGECO and Venus with a short, low-frequency ultrasound pulse $(0.5 \mathrm{MHz}$, all pulses consisted of 3-sec duration, 2000 cycles, $10 \mathrm{~Hz}, 0.5 \mathrm{MPa}$ unless otherwise noted). Live-cell imaging showed that a short ultrasound pulse of $0.5 \mathrm{MHz}$ was sufficient to evoke calcium influx in cells expressing Venus-mPrestin(N7T, N308S), but not in cells transfected with Venus alone (Fig. 1b; Extended Data Video 1). Quantification of the calcium imaging data indicated that FUS induced a $351 \pm 20 \%$ (mean \pm s.e.m.) increase in the R-GECO fluorescence of Venus-mPrestin(N7T, N308S)-transfected cells (Fig. 1c, right panel). However, FUS only slightly evoked the calcium response in cells that expressed Venus-mPrestinWT (Fig. 1c, middle panel). Cells transfected with Venus alone did not respond to FUS stimulation (Fig. 1c, left panel). These results indicated that heterogeneous expression of Venus-mPrestinWT endowed the transfected cells with a weak ability to sense ultrasound. Substituting Thr at position 7 and Ser at position 308 in the Venus-tagged mPrestinWT substantially improved the ultrasound-evoked calcium response of the transfected HEK293T cells.

To determine the optimal ultrasound frequency/frequencies for cell manipulation, we next comprehensively tested the calcium responses of cells expressing the various VenusmPrestin constructs to different FUS frequencies between $80 \mathrm{kHz}$ and $3.5 \mathrm{MHz}$ (3-sec duration, 2000 cycles, $0.5 \mathrm{MPa}$; Fig. 2). Interestingly, cells individually expressing the WT and mutated constructs were sensitive only to $0.5 \mathrm{MHz}$ FUS (Fig. 2). The $80 \mathrm{kHz}, 1 \mathrm{MHz}, 2 \mathrm{MHz}$, and 3.5 MHz FUS were insufficient to evoke a calcium influx in the cells (Fig. 2). Upon stimulation by $0.5 \mathrm{MHz}$ FUS, the percentage of ultrasound-responsive cells was $11.29 \pm 4.25$-fold (mean \pm s.e.m.) greater for the Venus-mPrestin(N7T, N308S) group compared with the control group ( $p=0.024$; Fig. 2). Heterogeneous expression of Venus-mPrestinWT, Venus-mPrestin(N7T), 
139

140

141

142

144

145

146

147

148

149

150

151

152

153

154

155

156

157

158

159

160

161

and Venus-mPrestin(N308S) only slightly increased the sensitivity of the transfected HEK293T cells to $0.5 \mathrm{MHz}$ FUS ( $p=0.31,0.51$, and 0.25 , respectively; Fig. 2). These results confirmed that $0.5 \mathrm{MHz}$ FUS efficiently evoked a calcium response in cells expressing mPrestin(N7T, N308S) in a frequency-dependent manner.

In addition to prestin, Ibsen and colleagues demonstrated that the mechanosensitive ion channel, TRP-4, is required for ultrasound-mediated mechanical stimulation and can modify animal behaviour in the presence of $\mathrm{MBs}^{16}$. To test whether TRP-4 can act as an ultrasoundresponsive protein, we transfected HEK293T cells with two members of the mammalian TRPC4 family including human TRPC4 $\alpha$ (hTRPC4 $\alpha$ ) and mouse TRPC4 $\beta$ (mTRPC4 $\beta)^{27,28}$. The calcium response of cells expressing hTRPC4 $\alpha$ or mTRPC4 $\beta$ tagged with CFP upon FUS stimulation of different frequencies was examined and quantified. The percentage of ultrasound-excitable cells in the in the mTRPC4 $\beta$-CFP group was $3.29 \pm 0.94$-fold (mean \pm s.e.m.) greater than the control group upon stimulation with $0.5 \mathrm{MHz}$ FUS ( $p=0.044$; Extended Data Fig. 2). Ultrasound of $80 \mathrm{kHz}, 1 \mathrm{MHz}, 2 \mathrm{MHz}$, and $3.5 \mathrm{MHz}$ was not sufficient to induce a calcium response in cells expressing mTRPC4 $\beta$-CFP. Thus, mTRPC4 $\beta$-CFP is only weakly sensitive to $0.5 \mathrm{MHz}$ FUS. Although its protein sequence is very similar to that of mTRPC4 $\beta$, hTRPC4 $\alpha$-CFP did not respond to the low-frequency ultrasound stimulation at all (Extended Data Fig. 2). Taken together, the comprehensive examination of ultrasound sensing in cells transfected with different putative ultrasound-responsive proteins shows that mPrestin(N7T, N308S) was the most responsive protein.

We next explored the possible molecular mechanisms that make the two evolutionarily conserved amino acid substitutions important for prestin-dependent ultrasound sensing. Targeting of prestin to the plasma membrane is required for its 
162 function ${ }^{29}$. Confocal images of Venus-mPrestinWT and Venus-mPrestin(N7T, N308S) in

163 living cells showed that Venus-mPrestinWT localised to the cytosol and to the plasma

164 membrane, whereas Venus-mPrestin(N7T, N308S) localised exclusively to the plasma

165 membrane (Fig. 3a). Quantification of the relative intensities confirmed that

166 mPrestin(N7T, N308S) exhibited a significantly greater plasma membrane/cytosol

167 intensity ratio than did Venus-mPrestinWT ( $p=0.003$; Fig. 3b). We therefore

168 hypothesised that targeting mPrestin(N7T, N308S) to the plasma membrane is important

169 for its sensitivity to ultrasound. To assess this hypothesis, we introduced a point mutation

170 (Y667Q) that causes prestin to mislocate to the Golgi apparatus into Venus-

171 mPrestin(N7T, N308S). As expected, Venus-mPrestin(N7T, N308S, Y667Q) accumulated

172 at the Golgi apparatus, and its plasma membrane/cytosol intensity ratio decreased

173 significantly ( $p=1.02 \mathrm{E}-7$; Fig. 3a, b). The mislocalisation of Venus-mPrestin(N7T, N308S,

174 Y667Q) to the Golgi apparatus impaired its ultrasound-sensing ability ( $p=0.032$; Fig. 3c),

175 confirming that plasma-membrane targeting of Venus-mPrestin(N7T, N308S) is required

176 for its response to ultrasound.

177 Venus-mPrestin(N7T, N308S) was not evenly distributed in the plasma membrane

178 but was concentrated in punctate regions (Figs. 1b and 3a). HEK293T cells expressing

179 mPrestin(N7T, N308S) exhibit significantly higher number of puncta than cells 180 expressing wild-type mPrestin ( $p=0.015$; Extended Data Fig. 3a). Mislocation of 181 mPrestin(N7T, N308S, Y667Q) at Golgi reduces the number of puncta suggesting puncta

182 formation of mPrestin requires its plasma membrane targeting ( $p=0.032$; Extended Data

183 Fig. 3a). Quantification data show the area of mPrestin(N7T, N308S) puncta is $132 \pm 6.28$

$184 \mathrm{~nm}^{2}$ (mean \pm s.e.m.; Extended Data Fig. 3b). Prestin self-assembles into oligomers to form 
185

186

187

188

189

190

191

192

193

194

195

196

197

198

199

200

201

202

203

204

205

206

207

bullet-shaped complexes in the plasma membrane ${ }^{30,31}$. To evaluate whether selfassociation of prestin occurred in these punctate regions, we used fluorescence resonance energy transfer (FRET) to examine the oligomerisation of Venus- and CFPtagged mPrestin constructs. A greater FRET efficiency was obtained in the punctate regions of cells expressing mPrestin(N7T, N308S) as compared with cells transfected with mPrestinWT ( $p=0.025$; Extended Data Fig. 3c, d), indicating that self-association of mPrestin(N7T, N308S) but not mPrestinWT occurred in the punctate regions. Immunofluorescence staining also showed that mPrestin(N7T, N308S) puncta associated with actin filaments and microtubules (Extended Data Fig. 3e). Next, ultrafast imaging of cells transfected with Venus-mPrestin(N7T, N308S) (imaging interval, $17 \mathrm{~ms}$ ) was used to observe the real-time behaviour of Venus-mPrestin(N7T, N308S) puncta upon FUS stimulation. Live-cell imaging and quantification showed that Venus-mPrestin(N7T, N308S) puncta oscillated continuously for a few seconds after being exposed to pulsed 0.5 MHz FUS (Fig. 3d, e; Extended Data Video 2). Because several waves of calcium responses were observed after a single FUS pulse in the Venus-mPrestin(N7T, N308S)-transfected cells (Fig. 1c, right), we hypothesised that a short pulse of FUS induced sustained oscillation of Venus-mPrestin(N7T, N308S)-positive puncta that then trigger the calcium response for a few seconds. To address this hypothesis, we found that cellular expression of Venus-mPrestin(N7T, N308S, V499G, Y501H), which prevents the electromotility of prestin without affecting its localisation to the plasma membrane ${ }^{32}$, blocked oscillation of the puncta upon FUS stimulation (Fig. 3d, e; Extended Data Video 2). Moreover, the lack of oscillation found for the Venus-mPrestin(N7T, N308S, V499G, Y501H) puncta significantly attenuated the FUS-mediated calcium response $(p=0.016$; 
208

209

210

211

212

213

214

215

216

217 the possible involvement of voltage-gated ion channels in our system, cells expressing

Fig. 3c). Thus FUS-evoked calcium responses are highly dependent on the electromotility and oscillation of prestin puncta in the plasma membrane.

We next determined in which cellular compartment the calcium is stored that is released by Venus-mPrestin(N7T, N308S) upon FUS stimulation. Addition of the calcium chelator ethylene glycol tetraacetic acid (EGTA) in the extracellular space completely inhibited the calcium response in cells expressing Venus-mPrestin(N7T, N308S) upon ultrasound stimulation ( $p=6.2 \mathrm{E}-7$; Fig. 3f). However, depletion of the intracellular calcium store by thapsigargin did not significantly affect the ultrasound-mediated calcium response ( $p=0.16$; Fig. 3f). Thus mPrestin(NT7, N308S) induced calcium influx from the extracellular space instead of from the intracellular calcium pool after FUS excitation. We speculate that replacement of Asn with Thr and Ser at positions 7 and 308, respectively, in mPrestin enhanced its localisation to the plasma membrane where its oscillations promoted calcium influx from the extracellular pool.

Several mechanosensitive ion channels are activated by high-frequency ultrasound 20,21 . We incubated gentamicin, a pharmaceutical inhibitor of mechanosensitive ion channels, with cells that expressed Venus-mPrestin(N7T, N308S) ${ }^{33}$ and found that this treatment did not significantly affect the calcium response upon ultrasound excitation ( $p=0.27$; Fig. 3f). Thus gentamicin-sensitive ion channels were not involved in the mPrestin(N7T, N308S)-mediated calcium response, which is consistent with results from an ultrasound-inducible system driven by piezoelectric nanoparticles ${ }^{34}$. Ultrasound excites neuronal cells by activating voltage-gated ion channels ${ }^{6}$. To examine Venus-mPrestin(N7T, N308S) were incubated with tetrodotoxin (TTX), an inhibitor of 
voltage-gated ion channels, and then stimulated with $0.5 \mathrm{MHz}$ FUS. However, the percentage of ultrasound-excitable cells transfected with mPrestin(N7T, N308S) was not affected by TTX treatment, indicating that voltage-gated ion channels are not involved in the mPrestin-mediated pathway ( $p=0.80$; Fig. 3f).

To take advantage of the great sensitivity of Venus-mPrestin(N7T, N308S) to ultrasound stimulation, we next developed a sonogenetic system that would allow for stimulating neurons (Fig. 4). Infection of primary cultured cortical neurons with a VenusmPrestin(N7T, N308S)-containing lentivirus led to the expression of VenusmPrestin(N7T, N308S) on a neuronal membrane. Moreover, Venus-mPrestin(N7T, stimulation of target neurons in deep brain, an adeno-associated virus (AAV) encoding Venus-mPrestin(N7T, N308S) or Venus alone was injected into the VTA brain region. Two weeks later, anesthetized mice were exposed to transcranial pulsed ultrasonic excitation (0.5 MHz FUS, $0.5 \mathrm{MPa}, 5$ seconds; Fig. 4b). FUS-activated neurons were mapped by imaging the expression of c-Fos (Fig. 4c). Neuronal excitation was triggered by a short pulsed FUS in Venus-mPrestin(N7T, N308S)-transfected mice ( $p=8.64 \mathrm{E}-3$,

247 Fig. 4c,d). Control mice with Venus alone expression showed no significant c-Fos 248 expression in VTA region ( $p=0.08$, Fig. $4 \mathrm{c}, \mathrm{d})$. These results demonstrated that mPrestin(N7T, N308S)-mediated sonogenetics is a flexible and non-invasive approach for sonogenetic control of neuronal activity.

In summary, we here have introduced two evolutionarily conserved amino acid substitutions N7T and N308S into mouse prestin which enhances its self-association as well as puncta formation in the plasma membrane. These mPrestin(N7T, N308S) puncta 
254 highly associate with actin filaments and microtubules in cells. A short pulse of $0.5 \mathrm{MHz}$

255 FUS induces sustained oscillation of mPrestin(N7T, N308S) puncta with electromotility

256

257

258

259

260

261

262

263

264

265

266

267

268

269

270

271

272

273

274

275

276 and evokes several waves of calcium responses in transfected cells (Extended Data Fig. 4). The ultrahigh ultrasound sensitivity of mPrestin(N7T, N308S) allows for noninvasively stimulation of target neurons in deep mice brain by a short pulsed FUS.

Our results raised a fundamental question: why are mPrestin(N7T, N308S)transfected cells sensitive only to ultrasound of 0.5 MHz? Because we used a short, lowfrequency, and low-pressure ultrasound pulse of constant acoustic power (0.5 MPa), it is unlikely that any unexpected thermal and/or mechanical effects were present that would restrict the frequency to $0.5 \mathrm{MHz}$. We therefore assume that a frequency of $0.5 \mathrm{MHz}$ is simply optimal for stimulation of cells. Cell membranes are able to absorb ultrasound waves and transient cavitation effect occurs in their intramembrane spaces upon ultrasound stimulation ${ }^{35}$. According to simulation and experimental data, $0.25 \sim 0.5 \mathrm{MHz}$ are the optimal frequencies of ultrasound for inducing intramembrane cavitation as well as bio-piezoelectric perturbation 7,36 . Because prestin acts as a piezoelectric amplifier to enhance the electromotile response in $\mathrm{OHCs}$ and mammalian cell lines ${ }^{25,26}$, we suggest that the ultrasound-induced intramembrane bio-piezoelectric perturbation may be amplified by mPrestin(N7T, N308S) that then trigger the observed calcium influx. Ultrasound of $80 \mathrm{kHz}$, which is the peak frequency used by most sonar species ${ }^{23}$, did not efficiently induce a calcium response in Venus-mPrestin(N7T, N308S)-transfected cells (Fig. 2), which suggested that the mechanism(s) of how sonar-responsive species hear ultrasound by auditory organs may not be the same as in our system.

Similar to photon-responsive-proteins and fluorescent proteins, which absorb 
277 distinct wavelengths of light and allow for multiplex imaging and optogenetics,

278 mPrestin(N7T, N308S) specifically responds to $0.5 \mathrm{MHz}$ FUS, suggesting that a multiple-

279 frequency system using ultrasound of 1-15 $\mathrm{MHz}$ can be developed to non-invasively 280 diagnose regions of abnormal tissues and simultaneously manipulate cellular activities with $0.5 \mathrm{MHz}$ FUS. Moreover, because 0.5 MHz FUS waves cannot be delivered through the air and are rarely used by sonar species, the natural background level for this frequency is expected to be low. Previously developed simulations and experimental data suggest that ultrasound wavelengths of $\sim 0.60-0.70 \mathrm{MHz}$ would be optimal for transcranial transmission and brain absorption 37,38 , supporting that our sonogenetic system is a promising tool for therapeutic applications involving the brain. Indeed, our in vivo results showed that $0.5 \mathrm{MHz}$ FUS efficiently accesses to the deep brain regions like VTA and stimulates target neurons expressing Venus-mPrestin(N7T, N308S) (Fig. 4c,d). To our knowledge, this mPrestin(N7T, N308S)-based sonogenetic approach is the first system that enables the use of low-frequency ultrasound to efficiently manipulate molecular activities in mammalian cells that are genetically modified. Although heterogeneous expression of mPrestin(N7T, N308S) significantly enhanced the ultrasound sensitivity of HEK293T cells $(p=0.0046,10.18 \pm 2.90 \%$ for the VenusmPrestin(N7T, N308S) group; $1.33 \pm 0.42 \%$ for the Venus-alone group; combined data shown in Figs. 1d and 2f), the percentage of ultrasound-excitable cells in our system needs improvement. A more detailed understanding of how mPrestin(N7T, N308S) sense and amplify ultrasound waves is needed to engineer different prestin variants that are more sensitive to ultrasound. With ongoing development, engineered ultrasound- 
tools for non-invasively and precisely manipulating activities of genetically modified cells.

301

302

\section{Online Methods}

303

304

305

306

307

308

309

310

311

312

313

314

315

316

317

\section{Cell culture, chemical reagents, DNA constructs, and transfection}

Human HEK293T cells were cultured in Dulbecco's modified Eagle's medium (DMEM;

Gibco) supplemented with $10 \%(\mathrm{v} / \mathrm{v})$ fetal bovine serum, $5 \mathrm{U} / \mathrm{ml}$ penicillin, and $50 \mu \mathrm{g} / \mathrm{ml}$ streptomycin (Gibco). The following Venus- or CFP-tagged mPrestin mutant genes were generated by site-directed mutagenesis: N7T, N308S, Y667Q, V449G, and Y501H. To construct the pLenti-hSyn1-Venus and pLenti-hSyn1-Venus-mPrestin(N7T, N308S), Q5® High-Fidelity DNA polymerase (New England Biolabs) and HiFi ${ }^{\circledR}$ assembly kit (New England Biolabs) were used. The hSyn1-Venus and hSyn1-Venus-mPrestin(N7T, N308S) inserts were PCR amplified from hSyn1-Venus-mPrestin(N7T, N308S) construct. The pLenti-backbone and the insert with a molar ratio of 1:2 (backbone:fragment) were $\mathrm{HiFi}{ }^{\circledR}$ assembled to acquire the corresponding lentiviral vectors. For DNA transfection, LT-1 (Mirus) was used according to the manufacturer's protocol. For inhibitor experiments, gentamicin (200 $\mu \mathrm{M}, 20 \mathrm{~min}$, Sigma), TTX (500 nM, $20 \mathrm{~min}$, Abcam), EGTA (5 mM, $20 \mathrm{~min}$, Sigma), and thapsigargin (100 nM, 30min, Sigma) were used. Before ultrasound excitation, HEK 293T cells were incubated with one of the various inhibitors or $0.1 \%$ (v/v) DMSO dissolved in DMEM (Gibco) at $37^{\circ} \mathrm{C}$. Calcium-free, serum-free medium (Gibco) was used in the EGTA experiment.

\section{Live-cell imaging}

Transfected cells were seeded into a Lab-Tek eight-well chambers (Thermo Scientific) coated with poly-D-lysine (P6407, Sigma-Aldrich) or onto 25-mm cover glasses in six-well 
323

324

325

326

327

328

329

330

331

332

333

334

335

336

337

338

339

340

341

342

culture plates (SPL Life Science) that were similarly coated. Live-cell imaging was conducted using a Nikon T1 inverted fluorescence microscope (Nikon) with a $20 \times$ or $60 \times$ oil objective (Nikon), a DS-Qi2 CMOS camera (Nikon), and Nikon element AR software (Nikon). The cells were held under a $5 \% \mathrm{CO}_{2}$ atmosphere at $37^{\circ} \mathrm{C}$ in an environmental chamber (Live Cell Instrument). The distribution of Venus-mPrestinWT, Venus-mPrestin(N7T, N308S), Venus-mPrestin(N7T, N308S, Y667Q), and Venus-mPrestin(N7T, N308S, V499G, Y501H) in HEK293T cells was observed using a Nikon A1 confocal system with a $100 \times$ oil objective (Nikon). Multiple $z$-stack images ( $0.3 \mu \mathrm{m}$ between stacks; 15 stacks) were acquired and processed with Huygens deconvolution (Scientific Volume Imaging), and the maximum intensity projections of the images were generated by Nikon element AR software. The plasma membrane/cytosol intensity ratios of the Venus-mPrestin constructs were analysed by Nikon element AR software. Ultrafast imaging was acquired under a Nikon A1 confocal system with a Resonant scanner (Nikon) and $100 \times$ objective (Nikon).

\section{Immunofluorescence staining}

HEK293T cells transfected with Venus-mPrestin(N7T, N308S) were seeded on poly-Dlysine-coated Lab-Tek eight-well chambers (Thermo Scientific). Transfected cells were fixed with 4\% paraformaldehyde (Electron Microscopy Sciences) at room temperature for 15 min and subsequently permeabilized by $0.1 \%$ Triton X-100 (Sigma-Aldrich). After incubation of blocking solution (PBS with $2 \%$ bovine serum albumin) for $30 \mathrm{~min}$ at room temperature, cells were stained with phalloidin Alexa Fluor 594 (1:100 dilution; Thermo Scientific, A12381) or anti- $\boldsymbol{\alpha}$-tubulin antibody (1:1000 dilution; Sigma-Aldrich, T6199) for $1 \mathrm{~h}$ at room temperature. 
346 Goat anti-mouse IgG Alexa Fluor 594 (1:1000 dilution; Thermo Scientific, R37121) were

347 incubated with cells for $1 \mathrm{~h}$ at room temperature.

348

\section{In vitro FUS stimulation}

FUS stimulation (acoustic power, 0.5 MPa; 2000 cycles; pulse repetition frequency, 10

Hz; and 3-sec duration) was applied using a single-element FUS transducer (Panametrics). The

ultrasound transducer was driven by a function generator (AFG3251, Tektronix) and a radio-

FUS: 325LA, Electronics \& Innovation)) to transmit the ultrasound pulses. A water cone filled the microscope. The transducer was calibrated in the free field in degassed water using a calibrated ultrasound power meter (Model UPM-DP-1AV, Ohmic Instruments Inc.).

\section{Lentivirus Production}

$5 \mathrm{~h}$ prior to transfection, culture medium of HEK293T cells grown to a confluency of $60 \%$ was replaced with $10 \mathrm{ml}$ DMEM supplemented with GlutaMAX (Gibco, Taipei, Taiwan) and 10\% FBS (Hyclone, Taipei, Taiwan) containing $25 \mu \mathrm{M}$ chloroquine diphosphate (Tokyo Chemical Industry, Taipei, Taiwan). HEK293T cells were co-transfected with 1.3 pmol psPAX2 (gift from Didier Trono; Addgene plasmid \# 12260), 0.72 pmol pMD2.G (gift from Didier Trono; 
369

370

371

372

373

374

375

376

377

378

379

380

381

382

383

384

385

386

387

MW25,000) transfection. The ratio of DNA:PEI was 1:3 diluted in $1 \mathrm{ml}$ of OptiMEM (Gibco).

$18 \mathrm{~h}$ post-transfection, viral medium was replaced with $15 \mathrm{ml}$ DMEM supplemented with

GlutaMAX and 10\% FBS. 48 h post-transfection, viral medium was harvested, stored at $4{ }^{\circ} \mathrm{C}$ and replaced with 15 ml DMEM supplemented with GlutaMAX and 10\% FBS. 72 h posttransfection, viral medium was pooled with the $48 \mathrm{~h}$ harvest, and centrifuged at $500 \mathrm{x} \mathrm{g}$ for 10 min at $4{ }^{\circ} \mathrm{C}$. The viral supernatant was filtered through $0.45 \mu \mathrm{m}$ PES filter (Pall, Taipei, Taiwan), snap frozen with liquid nitrogen, and stored at $-80^{\circ} \mathrm{C}$.

\section{Primary neuronal culture and lentivirus transduction}

Sprague-Dawley rats were purchased from BioLASCO Taiwan Co., Ltd. Primary cortical neurons were dissociated from dissected cortices of rat embryos (embryonic day 18, E18) and then seeded on poly-L-lysine (Sigma, Saint Louis, MO) -coated bottom-glass dishes $\left(1.2 \times 10^{6}\right.$ cells per dish). On day in vitro 0 (DIV0), primary neurons were cultured in Minimum Essential Medium (Invitrogen, Carlsbad, CA) supplemented with 5\% fetal bovine serum (Invitrogen), $5 \%$ horse serum (Invitrogen), and $0.5 \mathrm{mg} / \mathrm{ml}$ penicillin-streptomycin (Invitrogen) under 5\% $\mathrm{CO}_{2}$ condition. Culture medium was changed to Neurobasa $1^{\circledR}$ medium (Gibco, Grand Island, $\mathrm{NY}$ ) containing $25 \mu \mathrm{M}$ glutamate (Sigma), 2\% B-27 ${ }^{\mathrm{TM}}$ supplement (Invitrogen), $0.5 \mathrm{mM} \mathrm{L-}$ Glutamine (Invitrogen), and 50 units/ml Antibiotic-Antimycotic (AA) (Invitrogen) on DIV1. $10 \mu \mathrm{M}$ Cytosine- $\beta$-D-arabinofuranoside (AraC) (Invitrogen) was added to neurons on DIV2 to inhibit proliferation of glial cells. On DIV3, medium was changed to Neurobasal ${ }^{\circledR}$ medium containing 2\% B-27 ${ }^{\mathrm{TM}}$ supplement, $0.5 \mathrm{mM}$ L-Glutamine and 50 units/ml AA. On DIV6, conditional medium was harvested and half-replaced with fresh Neurobasal/ Glutamine culture medium. Neurons were infected with hSyn1-Venus or hSyn1-Venus-mPrestin(N7T, N308S)- 
392

393

394

395

396

397

398

399

400

401

402

403

404

405

406

407

408

409

410

411

412

413

414

containing lentivirus on DIV7. After overnight incubation at $37^{\circ} \mathrm{C}$, the virus-containing medium was replaced with conditional culture medium mixed with equal volume of fresh medium. For further maintenance, the medium was half-changed with fresh Neurobasal/ Glutamine culture medium every 2 days. After lentivirus infection for 7 days, neurons were imaged by a Nikon T1 inverted fluorescence microscope (Nikon).

\section{Adeno associated virusViral Delivery}

The Venus-mPrestin(N7T, N308S) or Venus alone-containing adeno-associated virus (AAV) were packaged by NTU CVT-LS-AAV core. A total of $1 \mu \mathrm{L}$ of AAV encoding VenusmPrestin(N7T, N308S) or Venus alone were transcranial injected into the left ventral tegmental area (VTA; bregma: - $3 \mathrm{~mm}$, left: $0.5 \mathrm{~mm}$, depth: $4.2 \mathrm{~mm}$ ). During the experiment, the animal was anesthetized with $2 \%$ isoflurane gas and immobilized on a stereotactic frame. After AAV injection for 2 weeks, the mice were simulated by FUS.

\section{In vivo sonogenetic stimulation of VTA}

The AAV transfected mice were randomly divided into four groups: (1) AAV encoding Venus-mPrestin(N7T, N308S) + 0.5 MHz FUS stimulation group; (2) AAV encoding VenusmPrestin(N7T, N308S) without FUS group(n=3); (3) AAV encoding Venus alone $+0.5 \mathrm{MHz}$ FUS stimulation group ( $\mathrm{n}=3$ ); and (4) AAV encoding Venus alone without ultrasound group(n=3). The $0.5 \mathrm{MHz}$ sonication was applied transcranially at the left brain with the acoustic pressure of $0.5 \mathrm{MPa}, 2,000$ cycles, and $10 \mathrm{~Hz}$ of pulse repetition frequency, sonication duration of $5 \mathrm{sec}$ and one sonication sites. During the experiment, the animal was anesthetized with $2 \%$ isoflurane gas and immobilized on a stereotactic frame. 


\section{Immunohistochemistry staining (IHC)}

417 The successful stimulation of mPrestin(N7T, N308S)-transfected cells was verified by c-

418 Fos IHC staining. ${ }^{39}$ The brains of mice were removed were sacrificed at $90 \mathrm{~min}$ after $0.5 \mathrm{MHz}$ 419 FUS stimulation. The brains were then sliced into $15-\mu \mathrm{m}$ sections and incubated into $5 \%$ goat serum and PBS for $1 \mathrm{~h}$ to block the endogenous proteins. The sections were then incubated in

421 primary rabbit anti-c-Fos antibody (1:1000; SYSY) in antibody diluent for overnight. The sections were then incubated for $1 \mathrm{~h}$ in Dylight 594 conjugated anti-rabbit secondary antibody (1:500; GeneTex) in antibody diluent followed by several washes in PBS. The cellular nuclei medium and stored flat in the dark at $-20^{\circ} \mathrm{C}$. The successful transfection of pPrestin was confirmed by the expression of Venus fluorescence protein.

We analyzed the overlap between Venus tagged proteins (Venus alone or VenusmPrestin(N7T, N308S) and c-Fos by calculating the number of Venus positive cells and

Venus/c-Fos double positive cells in different animal groups. Means and s.e.m. were calculated across animals, and all statistics were done across animals.

\section{Acknowledgments}

434 PrestinWT construct; Dr. Insuk So (Seoul National University College of Medicine) for hTRPC4 $\alpha$-CFP and mTRPC4 $\beta$-CFP constructs; and Dr. Takanari Inoue (Johns Hopkins

436 University School of Medicine) for the CFP-R-GECO, R-GECO, mCherry-CAAX, and 437 mCherry-Giantin constructs. Dr. Takananri Inoue (Johns Hopkins University School of 
E-007-055 and 105-2119-M-182-001 to C.K.Y. 104-2320-B-007-005-MY2, and 106-2320-B-

001, and 108-2636-B-007-003 to Y.C.L.). Additional funding consisted of a grant from the

Program for Translational Innovation of Biopharmaceutical Development-Technology

iMATE-107-33) to C.P.L., and a grant from National Health Research Institutes (grant number

\section{Author Contributions}

Y.S.H., C.H.F., and N.H. contributed equally to this work. Y.S.H., C.H.F., C.K.Y., and Yu-Chun

Lin designed the experiments. C.H.F. and C.Y.W. programmed the ultrasound system, under biology experiments. Y.S.H., C.H.F., N.H., Y.C.Chang, Y.C.Chiang, and W.C.H. quantified the constructs. A.Y.W. designed and cloned the lentiviral plasmid, V.G. packaged the lentiviruses, the primary cultured cortical neurons under the supervision of L.C. C.H.F. performed the animal experiments. Y.S.H., C.H.F., C.K.Y., and Yu-Chun Lin wrote the paper. 
463 The patents of mPresin(N7T, N308S) and relative sonogenetic tools are pending.

Supplementary Information is available in the online version of the paper.

467

468

\section{References}

469 1. Yizhar, O., Fenno, L. E., Davidson, T. J., Mogri, M. \& Deisseroth, K. Optogenetics in Neural Systems. Neuron 71, 9-34 (2011).

2. Sternson, S. M. \& Roth, B. L. Chemogenetic Tools to Interrogate Brain Functions. Annu. Rev. Neurosci. 387-407 (2014). doi:10.1146/annurev-neuro-071013-014048

3. Qin, S. et al. A magnetic protein biocompass. Nat. Mater. 15, 217-226 (2015). regulation of glucose homeostasis in mice using genetically encoded nanoparticles. Nat. Med. 21, 92-98 (2015).

5. Maresca, D. et al. Biomolecular Ultrasound and Sonogenetics. Annu. Rev. Chem. Biomol. Eng. 9, annurev-chembioeng-060817-084034 (2018).

6. Tyler, W. J. et al. Remote excitation of neuronal circuits using low-intensity, lowfrequency ultrasound. PLoS One 3, (2008). 
484

485

486

487

488

489

490

491

492

493

494

495

496

497

498

499

500

501

502

503

504

505

506

8. Tufail, Y. et al. Transcranial Pulsed Ultrasound Stimulates Intact Brain Circuits. Neuron 66, 681-694 (2010).

9. Tufail, Y., Yoshihiro, A., Pati, S., Li, M. M. \& Tyler, W. J. Ultrasonic neuromodulation by brain stimulation with transcranial ultrasound. Nat. Protoc. 6, 1453-1470 (2011).

10. Yoo, S. S. et al. Focused ultrasound modulates region-specific brain activity. Neuroimage 56, 1267-1275 (2011).

11. Menz, M. D., Oralkan, O., Khuri-Yakub, P. T. \& Baccus, S. a. Precise neural stimulation in the retina using focused ultrasound. J. Neurosci. 33, 4550-4560 (2013).

12. Tsui, P. H., Wang, S. H. \& Huang, C. C. In vitro effects of ultrasound with different energies on the conduction properties of neural tissue. Ultrasonics 43, 560-565 (2005).

13. Foley, J. L. J., Little, J. W. J. J. W. \& Vaezy, S. Image-guided high-intensity focused ultrasound for conduction block of peripheral nerves. Ann. Biomed. Eng. 35, 109-119 (2007).

14. Meijering, B. D. M. et al. Ultrasound and microbubble-targeted delivery of macromolecules is regulated by induction of endocytosis and pore formation. Circ. Res. 104, 679-687 (2009).

15. Fan, Z., Liu, H., Mayer, M. \& Deng, C. X. Spatiotemporally controlled single cell sonoporation. Proc. Natl. Acad. Sci. 109, 16486-16491 (2012).

16. Ibsen, S., Tong, A., Schutt, C., Esener, S. \& Chalasani, S. H. Sonogenetics is a noninvasive approach to activating neurons in Caenorhabditis elegans. Nat. Commun. 6, $1-12(2015)$.

17. Dynamic, W. B. et al. Targeted Microbubbles for Imaging Tumor Angiogenesis : 
Assessment of Purpose : Methods : Results : Conclusion : 249, (2008).

508

509

510

511

512

513

514

515

516

517

518

519

520

521

522

523

524

525

526

527

528

529

18. Lakshmanan, A. et al. Molecular Engineering of Acoustic Protein Nanostructures. ACS Nano 10, 7314-7322 (2016).

19. Bourdeau, R. et al. Acoustic reporter genes for non-invasive imaging of microbes in mammalian hosts. Nat. Publ. Gr. 553, 86-90 (2018).

20. Prieto, M. L., Firouzi, K., Khuri-Yakub, B. T. \& Maduke, M. Activation of Piezo1 but Not NaV1.2 Channels by Ultrasound at 43 MHz. Ultrasound Med. Biol. 44, 1217$1232(2018)$

21. Ye, J. et al. [ASAP] Ultrasonic Control of Neural Activity through Activation of the Mechanosensitive Channel MscL. Nano Lett. (2018). doi:10.1021/acs.nanolett.8b00935

22. Fettiplace, R. \& Hackney, C. M. The sensory and motor roles of auditory hair cells. Nat. Rev. Neurosci. 7, 19-29 (2006).

23. Rossiter, S. J., Zhang, S. \& Liu, Y. Prestin and high frequency hearing in mammals. Commun. Integr. Biol. (2011). doi:10.4161/cib.4.2.14647

24. Liu, Z., Qi, F.-Y., Zhou, X., Ren, H.-Q. \& Shi, P. Parallel Sites Implicate Functional Convergence of the Hearing Gene Prestin among Echolocating Mammals. Mol. Biol. Evol. 31, 2415-2424 (2014).

25. Dallos, P. \& Fakler, B. Prestin, a new type of motor protein. Nat. Rev. Mol. Cell Biol. 3, 104-111 (2002).

26. Ludwig, J. et al. Reciprocal electromechanical properties of rat prestin: the motor molecule from rat outer hair cells. Proc. Natl. Acad. Sci. U. S. A. 98, 4178-4183 (2001). 
530

531

532

533

27. Song, H. B., Jun, H. O., Kim, J. H., Fruttiger, M. \& Kim, J. H. Suppression of transient receptor potential canonical channel 4 inhibits vascular endothelial growth factorinduced retinal neovascularization. Cell Calcium 57, 101-108 (2015).

28. Myeong, J., Kwak, M., Hong, C., Jeon, J. H. \& So, I. Identification of a membranetargeting domain of the transient receptor potential canonical (TRPC) 4 channel unrelated to its formation of a tetrameric structure. J. Biol. Chem. 289, 34990-35002 (2014).

29. Zhang, Y., Moeini-Naghani, I., Bai, J., Santos-Sacchi, J. \& Navaratnam, D. S. Tyrosine motifs are required for prestin basolateral membrane targeting. Biol. Open 197-205 (2015). doi:10.1242/bio.201410629

30. Greeson, J. N., Organ, L. E., Pereira, F. a. \& Raphael, R. M. Assessment of prestin self-association using fluorescence resonance energy transfer. Brain Res. 1091, 140150 (2006).

31. Mio, K. et al. The motor protein prestin is a bullet-shaped molecule with inner cavities. J. Biol. Chem. 283, 1137-1145 (2008).

32. Zheng, J. et al. The C-terminus of prestin influences nonlinear capacitance and plasma membrane targeting. J. Cell Sci. 118, 2987-2996 (2005).

33. Jacques-Fricke, B. T. Ca2+ Influx through Mechanosensitive Channels Inhibits Neurite Outgrowth in Opposition to Other Influx Pathways and Release from Intracellular Stores. J. Neurosci. 26, 5656-5664 (2006).

34. Marino, A. et al. Piezoelectric Nanoparticle-Assisted Wireless Neuronal Stimulation. ACS Nano 9, 7678-7689 (2015).

35. Krasovitski, B., Frenkel, V., Shoham, S. \& Kimmel, E. Intramembrane cavitation as a 

3258-3263 (2011).

36. Plaksin, M., Shoham, S. \& Kimmel, E. Intramembrane cavitation as a predictive biopiezoelectric mechanism for ultrasonic brain stimulation. Phys. Rev. X 4, 1-10 (2014).

37. White, P. J., Clement, G. T. \& Hynynen, K. Local frequency dependence in transcranial ultrasound transmission. AIP Conf. Proc. 829, 256-260 (2006).

38. Hayner, M. \& Hynynen, K. Numerical analysis of ultrasonic transmission and 3319-3330 (2001).

Figure legends

responsive protein. (a) Sequence alignment of prestins from six non-echolocating and eight echolocating species showing that N7T and N308S substitutions frequently occurred in the echolocating species. Positions 7 and 308 are boxed with the residues located at those positions highlighted in red. (b) Excitation of 0.5 MHz FUS evokes calcium responses in cells expressing Venus-mPrestin(N7T, N308S) but not in control cells expressing Venus alone. Cells were cotransfected with the calcium biosensor CFP-R-GECO. The intensity of the R-GECO

571 fluorescence in the cells was monitored by live-cell imaging. Scale bar, $10 \mu \mathrm{m}$. (c) Time course

572 of R-GECO fluorescence intensity in cells expressing the indicated constructs before and after 573 the $0.5 \mathrm{MHz}$ FUS as described above. ATP treatment $(10 \mu \mathrm{M})$ served as a positive control to 574 show that the cells could exhibit intracellular calcium flux. Data were collected for 7-36 575 independent experiments, with $n=250$ cells per experiment. 
577 Figure $2 \mid$ mPrestin(N7T, N308S) enabled an ultrasound-evoked calcium response in a

578 frequency-specific manner. HEK293T cells transfected with one of the indicated DNA

579 constructs were bathed in PBS and stimulated with ultrasound of different frequencies (3-sec

580 duration, 2000 cycles, $0.5 \mathrm{MPa}$ ). Data are presented as the relative percentage of cells in each

581 group (expressed as fold-probability) that were excitable by ultrasound after normalisation to

582 that of cells expressing only Venus that were stimulated at the same frequency. The absolute

583 number of cells in each group was $998,556,686,739,780,3111,438,277,691,1484,1515$,

$584408,472,785,771,1735,856,1571,1085,520,1470,1050,1250,1199$, and 605 cells from

585 left to right. Data are shown as the mean \pm s.e.m. for 7-36 independent experiments. $P$-values

$586>0.05$ are not shown.

587

588

Figure 3 | mPrestin(N7T, N308S) puncta oscillate upon FUS stimulation and trigger

calcium influx from extracellular pool. (a) Representative confocal images of HEK293T

cells expressing Venus-mPrestinWT, Venus-mPrestin(N7T, N308S), mCherry-CAAX (a

591 plasma membrane marker), or mCherry-Giantin (a Golgi marker). For each field, the maximum

593 Quantification of the plasma membrane/cytosol ratio for the indicated mPrestin constructs.

594 Data are shown as the mean \pm s.e.m. for three independent experiments; $n=22$, 26, and 61

595 cells from left to right. (c) HEK293T cells expressing the indicated constructs were stimulated with 0.5 MHz FUS (3-sec duration, 2000 cycles, $0.5 \mathrm{MPa}$ ). Data are presented as in Figure 2. to right. The data are shown as the mean \pm s.e.m. for 6-36 independent experiments. (d) Video 
Figure 4 | Transcranial FUS stimulation of neuron in mice brain via mPrestin(NT, NS) different conditions. Extensive FUS-driven c-Fos (red) expression was detected in cells

616 Fos + Venus + cells. Scale bar, $100 \mu \mathrm{m}$. (d) Percentage of c-Fos-positive neurons expressing

617 Venus alone or Venus-mPrestin(N7T, N308S) with or without FUS stimulation. Data are shown as the mean \pm s.e.m. for $6 \sim 9$ different sections from 4 mice per condition. 
622

623

624

625

626

627

628

629

630

631

632

633

634

635

636

637

638

639

640

641

642

643

644

was placed in the medium of a culture dish containing a monolayer of cells for FUS excitation.

The behaviour of cells upon FUS stimulation in real time was observed through an inverted microscope.

\section{Extended Data Figure 2 | mTRPC4 $\beta$ enabled a weak ultrasound-evoked calcium response}

in a frequency-specific manner. HEK293 cells transfected with one of the indicated DNA constructs were bathed in PBS and stimulated with ultrasound of different frequencies (3-sec duration, 2000 cycles, $0.5 \mathrm{MPa}$ ). Data are presented as the relative number of cells in each group (expressed as fold-probability) that were excitable by ultrasound after normalisation to that of cells expressing only Venus that were stimulated at the same frequency. The absolute number of cells in each group was $1209,768,889,1634,1665,1736,1054,1035,1116,1012$, $1000,960,1168,857$, and 909 cells from left to right. Data are shown as the mean \pm s.e.m. for $7-17$ independent experiments. $P$ values $>0.05$ are not shown.

\section{Extended Data Figure 3 | Characterization of mPrestin(N7T, N308S)-positive puncta. (a)}

The average number of mPrestin-positive puncta in cells expressing the indicated constructs.

The number of cells counted in each group are 7, 3, and 3 cells from 3 independent experiments.

Data are shown as mean \pm s.e.m. (b) Size distribution of mPrestin(N7T, N308S)-positive puncta. $\mathrm{n}=101$ puncta from five cells expressing mPrestin(N7T, N308S). (c) HEK293 cells transfected with the indicated DNA constructs were imaged by fluorescence resonance energy transfer (FRET). Scale bars, $10 \mu \mathrm{m}$. (d) Quantification of the FRET/CFP ratios for cells expressing the indicated DNA constructs. The numbers of cells were 25 (mPrestinWT) and 21 (mPrestin(N7T, N308S). Data are shown as the mean \pm s.e.m. for two independent experiments. 
645 (e) HEK293T cells expressing Venus-mPrestin(N7T, N308S) were processed for 646 immunofluorescence with phalloidin (actin filaments) or anti- $\boldsymbol{\alpha}$-tubulin antibody

647 (microtubules). For each field, a maximal z projection was created form 15 stacks separated by $648 \quad 0.3 \mu \mathrm{m}$. Scale bar $=10 \mu \mathrm{m}$.

649

650 Extended Data Figure 4 | The working model of mPrestin(N7T, N308S)-mediated calcium

651 influx upon ultrasound stimulation. Two evolutionarily conserved mutants N7T and N308S

652 enhance self-assembly of mPrestin in the punctate regions of plasma membrane where they 653 associate with actin filaments and microtubules. The mPrestin(N7T, N308S)-positive puncta 654 with electromotility are oscillated upon $0.5 \mathrm{MHz}$ FUS stimulation (3-sec duration, 2000 cycles, $655 \quad 0.5 \mathrm{MPa}$ ) which triggers the influx of calcium from extracellular space.

656

657 Extended Data Video 1 | mPrestin(N7T, N308S) enables ultrasound-evoked calcium 658 response. Excitation of $0.5 \mathrm{MHz}$ FUS evokes calcium response in cells expressing Venus659 mPrestin(N7T, N308S) but not in control cells (Venus alone). The cells co-transfected with a 660 calcium biosensor, CFP-R-GECO, and Venus alone or Venus-mPrestin(N7T, N308S), were 661 excited by $0.5 \mathrm{MHz}$ pulsed FUS (3 sec duration, 2000 cycles, $0.5 \mathrm{MPa}$ ). The intensity of R-

662 GECO in cells was monitored by live-cell imaging. Scale bar $=10 \mu \mathrm{m}$.

663

Extended Data Video 2 | mPrestin(N7T, N308S)-positive puncta oscillated upon FUS stimulation. HEK293T cells were transfected with Venus-mPrestin(N7T, N308S) or VenusmPrestin(N7T, N308S, V499G, Y501H). Video showing the structural dynamics of mPrestin-

667 positive puncta in cells that had or had not been stimulated with $0.5 \mathrm{MHz}$ FUS. The boundaries 
bioRxiv preprint doi: https://doi.org/10.1101/625533; this version posted May 3, 2019. The copyright holder for this preprint (which was not certified by peer review) is the author/funder. All rights reserved. No reuse allowed without permission.

668 of the punctate regions are outlined in white. Scale bar $=0.2 \mu \mathrm{m}$.

669 


\section{Figure 1}

\begin{tabular}{|c|c|c|c|c|}
\hline \multirow[t]{2}{*}{$\mathbf{a}$} & Homo sapiens & 1 MDHAEENE I LAATQRYYVER $20 \ldots \ldots \ldots$ & 301 TGISAGF & LKESYNVDVVG 319 \\
\hline & Mus musculus & 1 MDHAE EN E I PAE TQRYYVER $20 \ldots \ldots \ldots$ & 301 TG I SAGFN & LHESYSVDVVG 319 \\
\hline \multirow{7}{*}{$\begin{array}{l}\text { Non- } \\
\text { echolocating } \\
\text { species }\end{array}$} & Pteropus vampyurs & 1 MDHAE ENE I LAATQRYYVER $20 \ldots \ldots \ldots$ & 301 TGISAGFN & LHESYNVDVVG 319 \\
\hline & Balaenoptera acutorostrata & ENE I LAAAQRYYVER $20 \ldots \ldots \ldots$ & 301 TG I SAGFN & LNESYNVDVVG 319 \\
\hline & Eonycteris spelaea & E ENE I LAATQRYYVER $20 \ldots \ldots \ldots$ & 301 TG I SAGFN & LHESYNVDVVG 319 \\
\hline & Rousettus leschenaultia & 1 MDHAE ENE I LAA TQRYYVER $20 \ldots \ldots \ldots$ & 301 TG I SAGFN & LHESYNVDVVG 319 \\
\hline & Phocoena phocoena & 1 MDHVEESE I LAA TQRYYVER $20 \ldots \ldots \ldots$ & 301 TG I SAGFS & LHESYNVDVVG 319 \\
\hline & Megaderma spasma & 1 MDHAE ETE I LAA TQKYYVER $20 \ldots \ldots \ldots$ & 301 TG I SAG & LHESYNVDVVG 319 \\
\hline & Megaderma lyra & 1 MDHAEETE I LAA TQKYYVER $20 \ldots \ldots \ldots$ & 301 TG I SAGFS & LHES YNVDVVG 319 \\
\hline \multirow{5}{*}{$\begin{array}{l}\text { Echolocating } \\
\text { species }\end{array}$} & Hyperoodon ampullatus & 1 MDHVEETE I LAATQRYYVER $20 \ldots \ldots \ldots .$. & 301 TG I SAGFN & LHESYNVDVVG 319 \\
\hline & Ziphius cavirostris & 1 MDHVEETE I LAA TQRYYVER $20 \ldots \ldots \ldots$ & 301 TG I SAGFN & LHES YNVDVVG 319 \\
\hline & Miniopterus fuliginosus & 1 MDHAEETE I LAA TQRYCVDR $20 \cdots \cdots \cdots$ & 301 TG I S AGFS & LHESYNVDVVG 319 \\
\hline & Tursiops truncatus & 1 MDHVEETE I LAATQRYYVER $20 \ldots \ldots \ldots$ & 301 TG I SAGFS & LHESYNVDVVG 319 \\
\hline & Myotis ricketti & 1 MDHAE ETE I LAAAQKYYVDR $20 \ldots \ldots \ldots$ & 301 TG I SAC & LHESYNVDVVG 319 \\
\hline
\end{tabular}

b

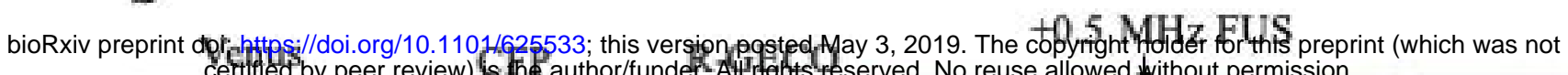
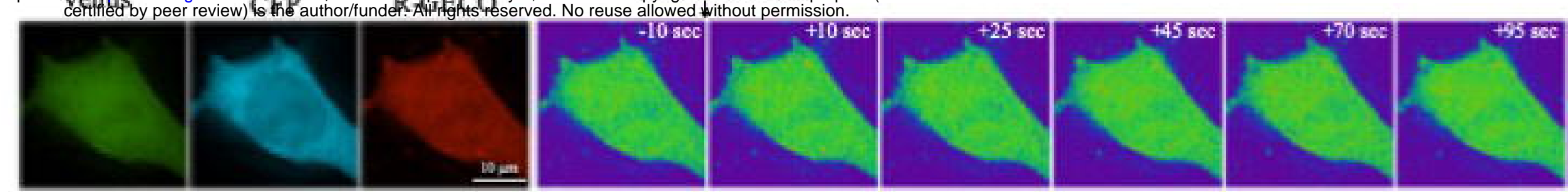

$$
\text { Venus: }
$$

mPrestin
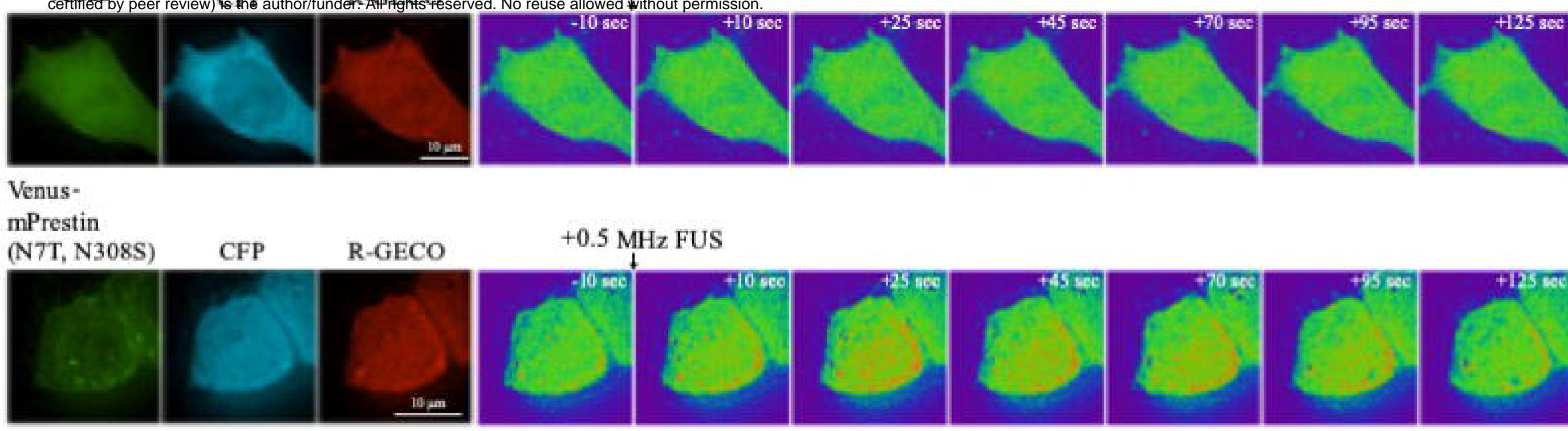

$$
\text { c }
$$

$0.5 \mathrm{MHz}$ Venus only FUS

$0.5 \mathrm{MHz}$ Venus-mPrestinWT FUS

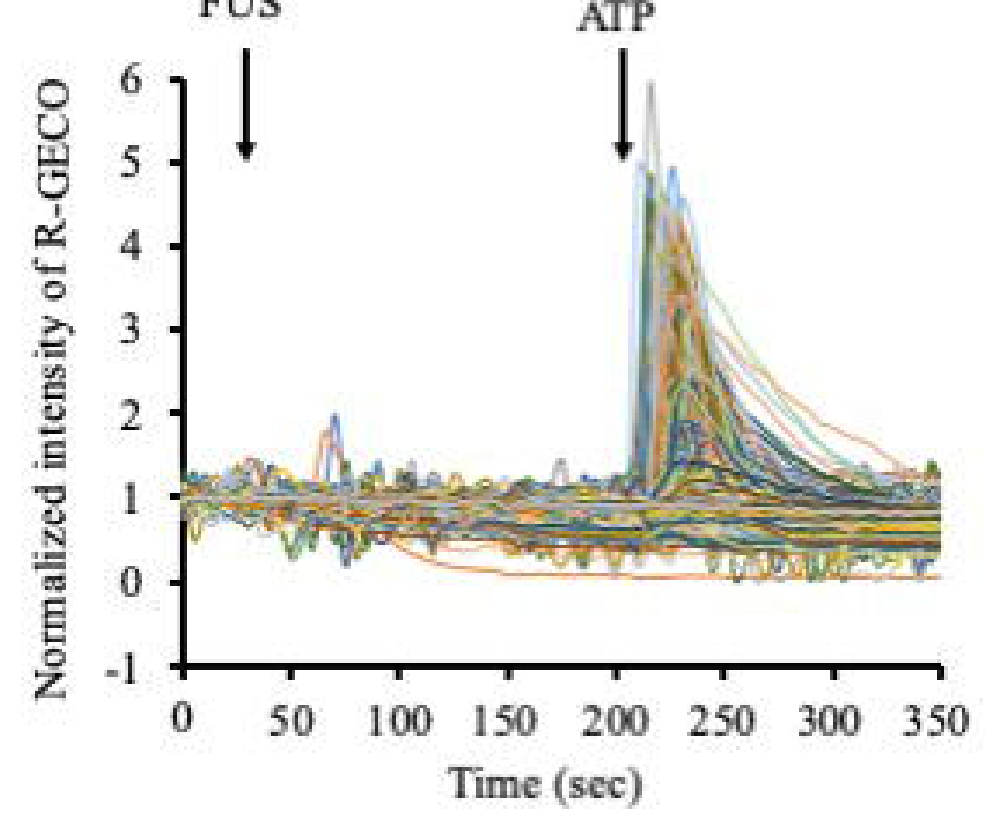

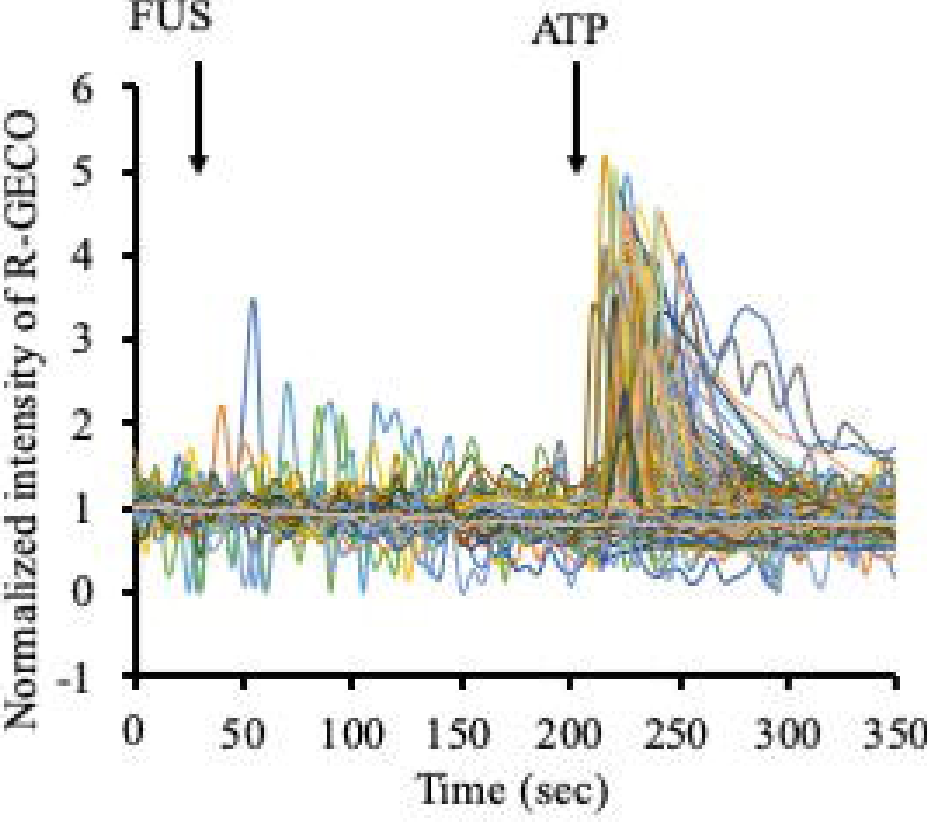

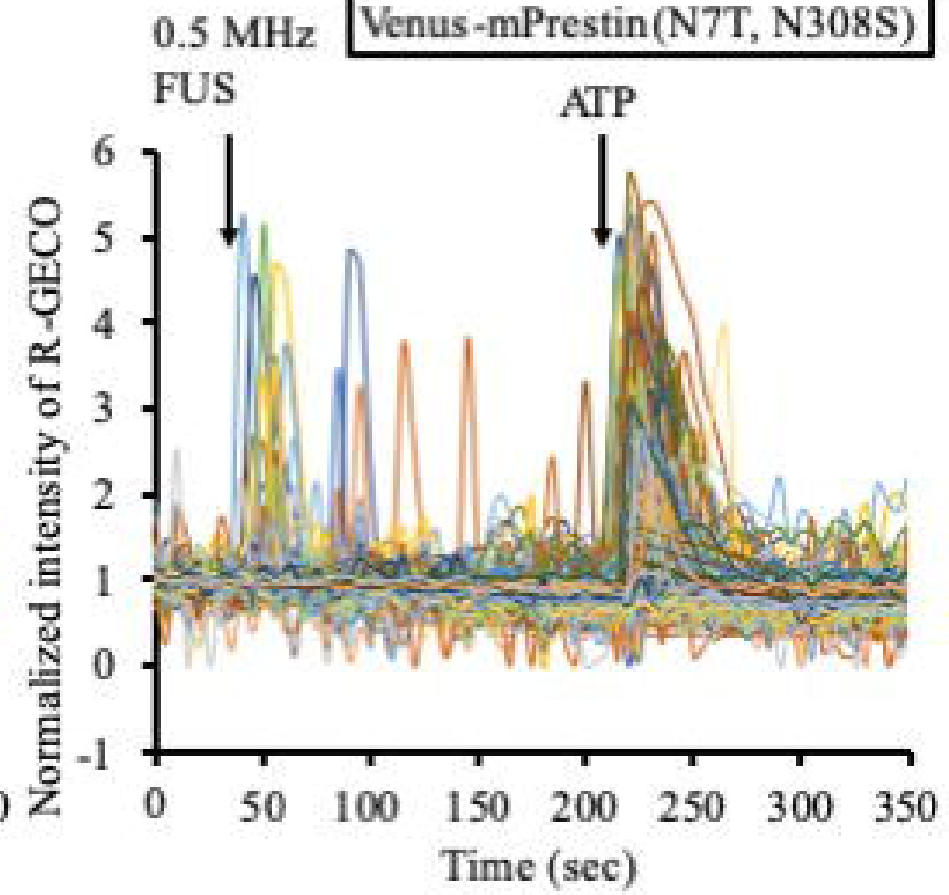


Figure 2

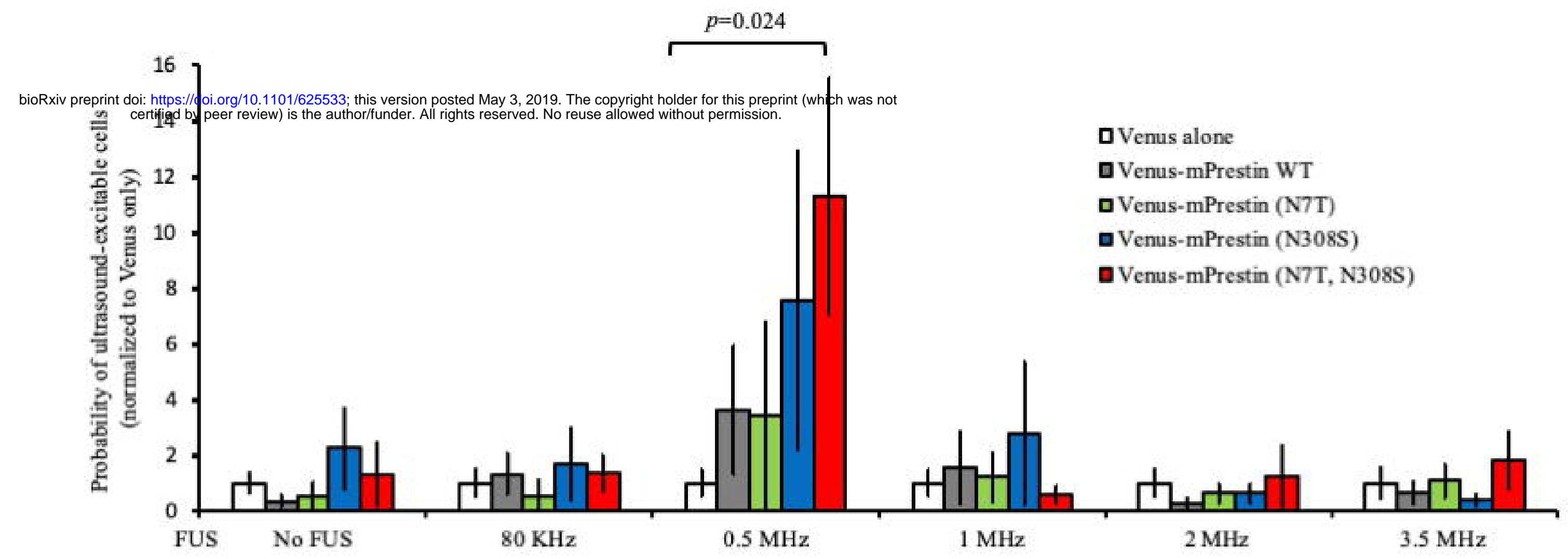




\section{Figure 3}

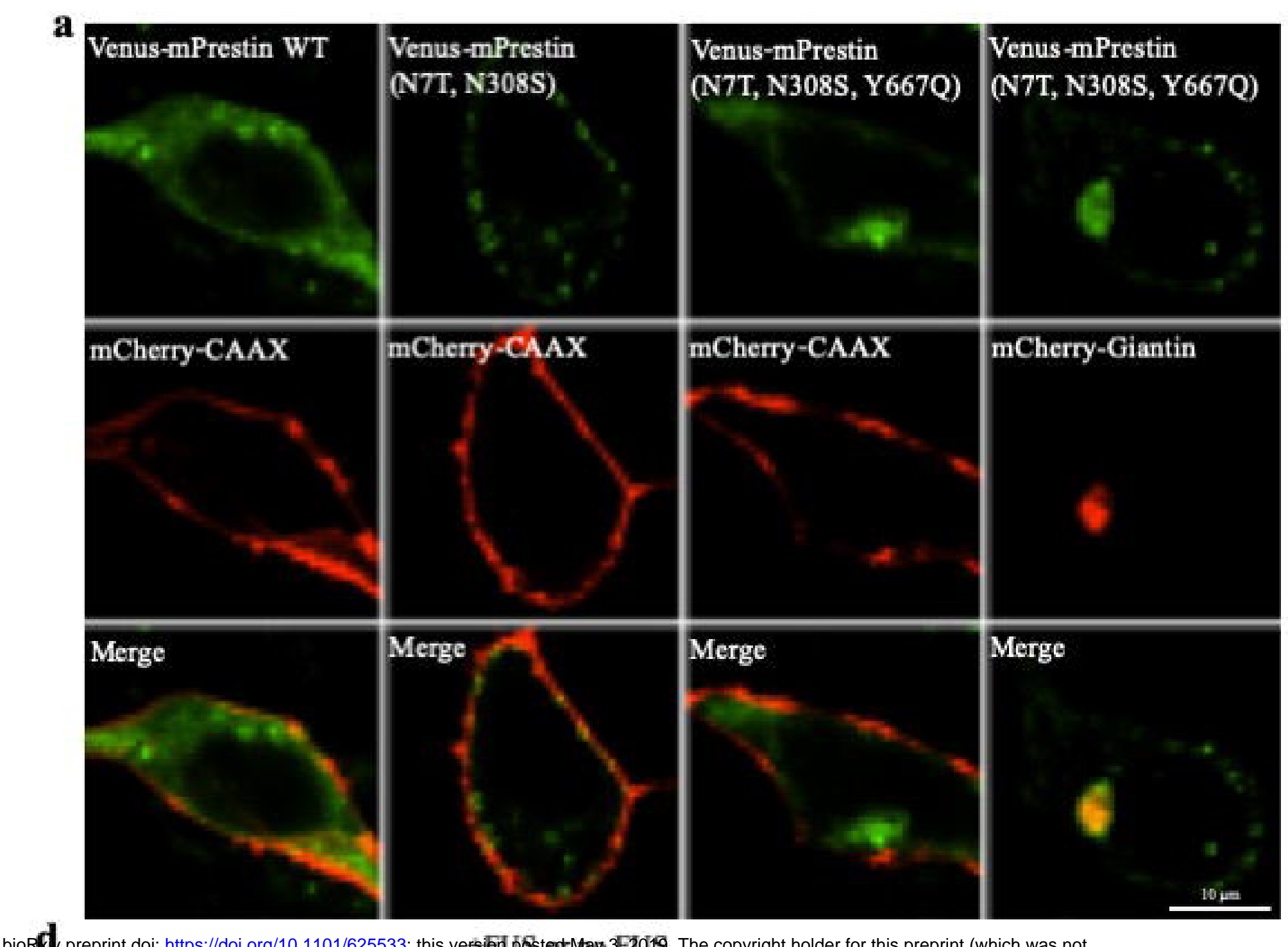

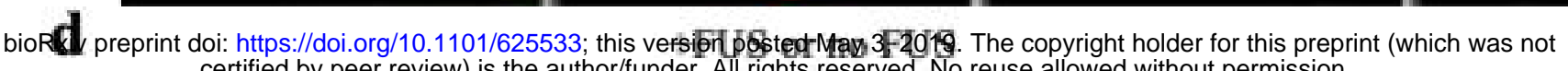
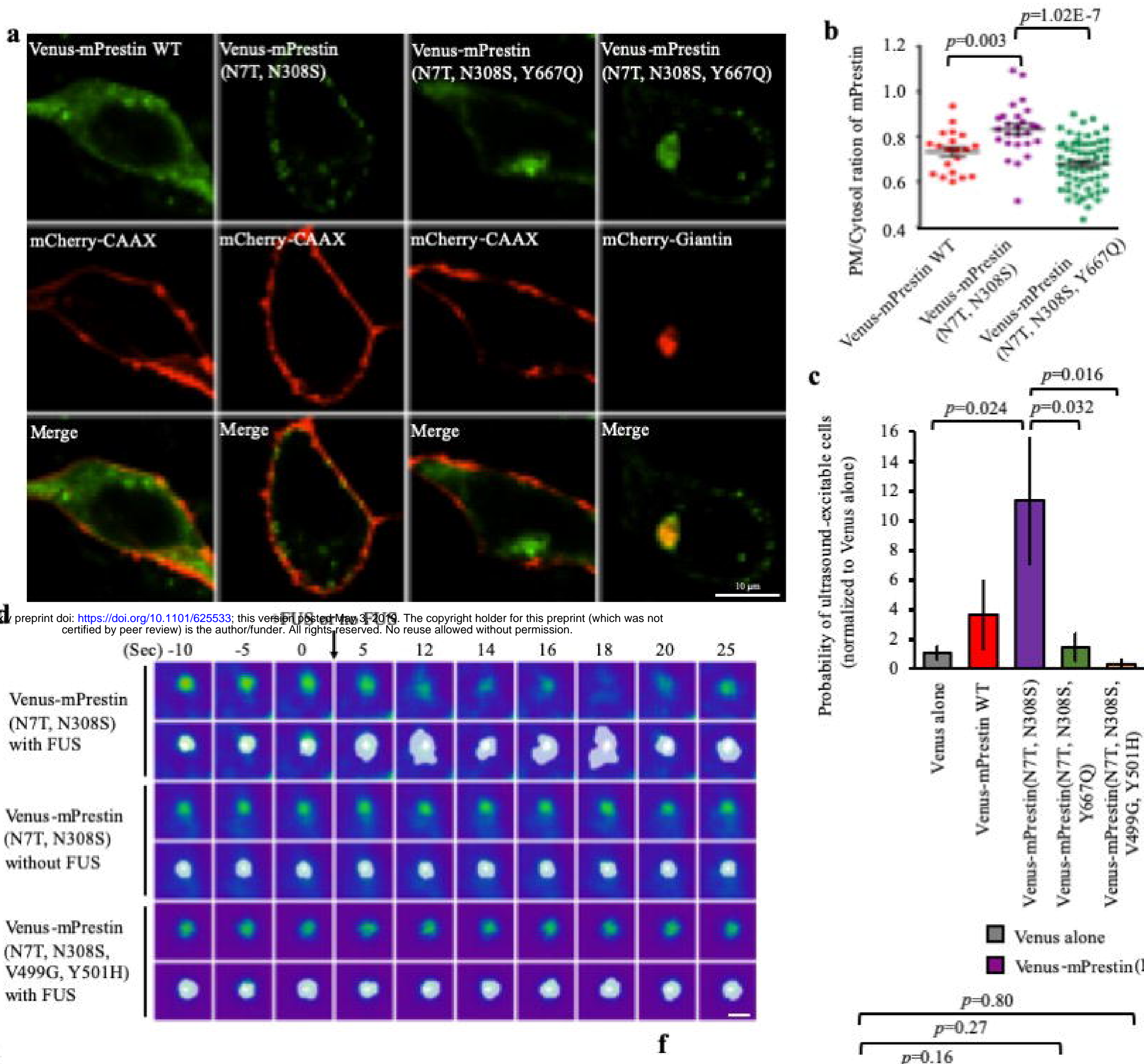

Venus-mPrestin (N7T, N308S, V499G, Y501H) with FUS

e
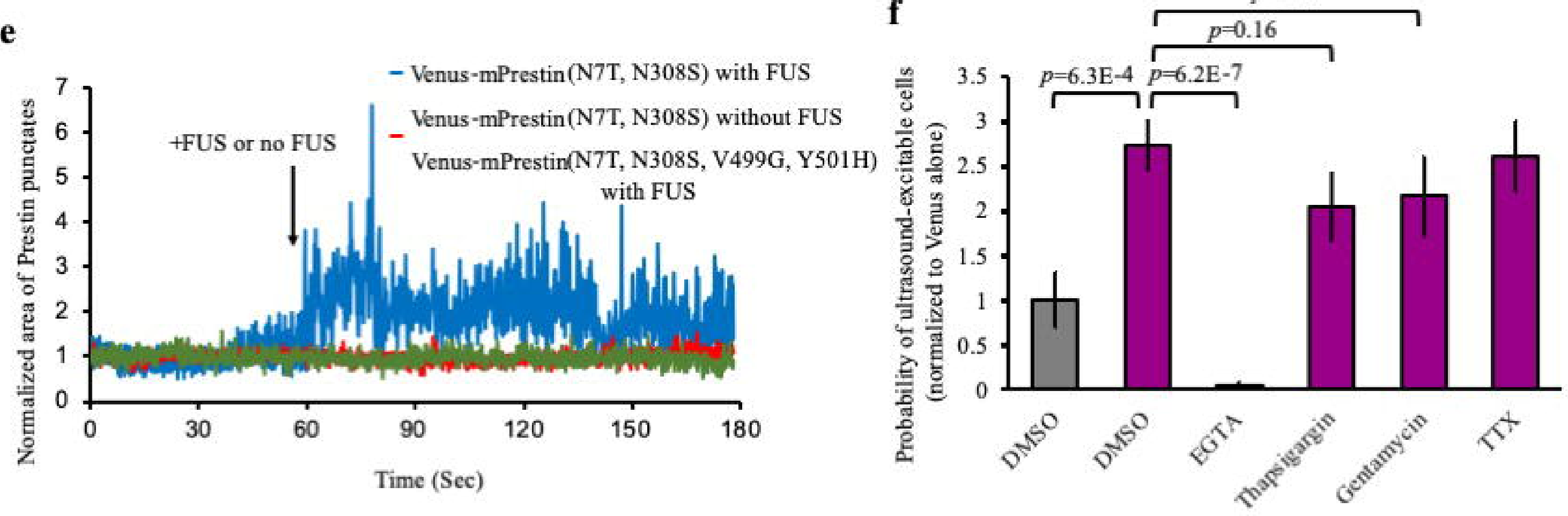


\section{Figure 4}

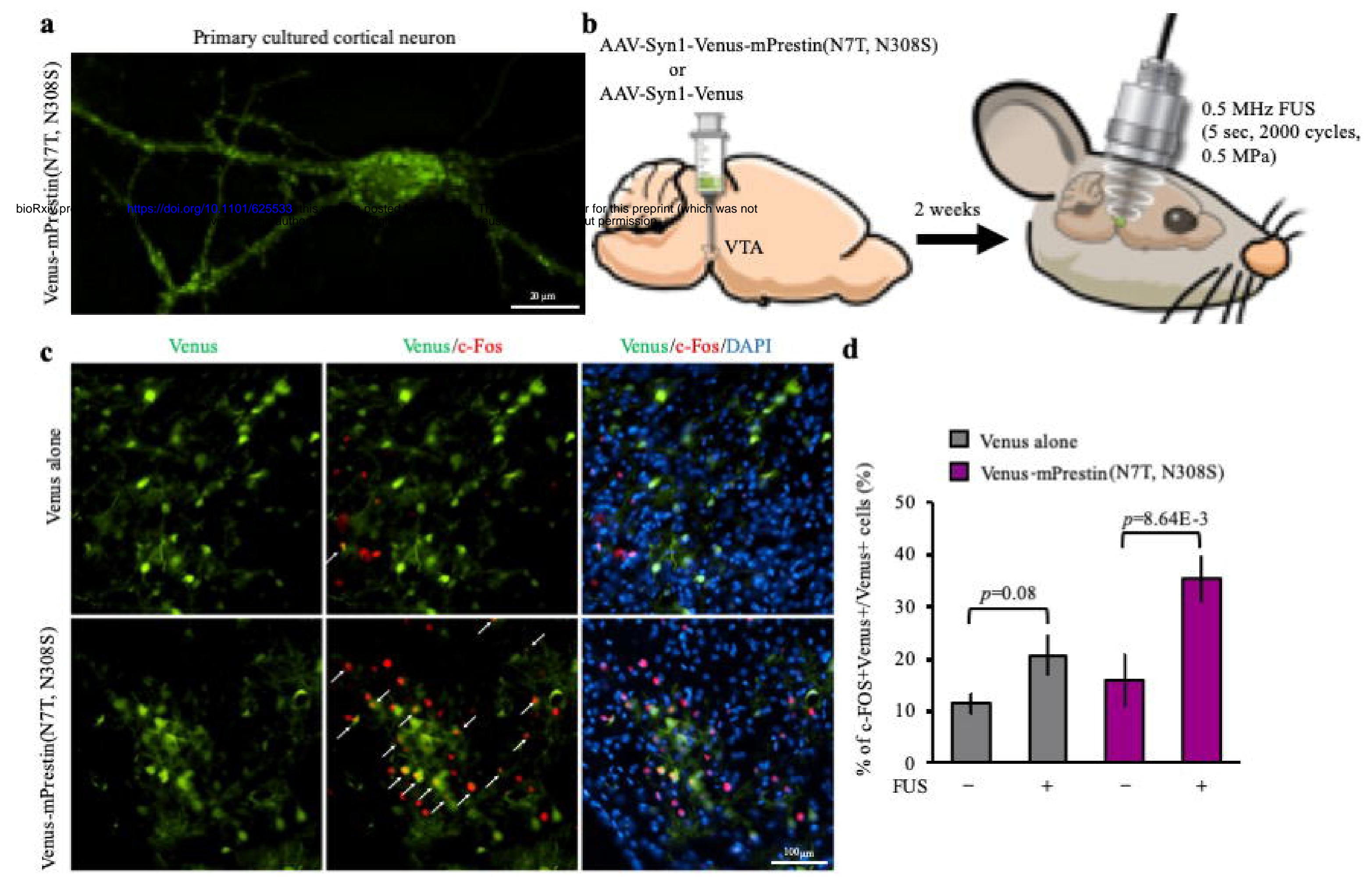




\section{Extended Figure 1}

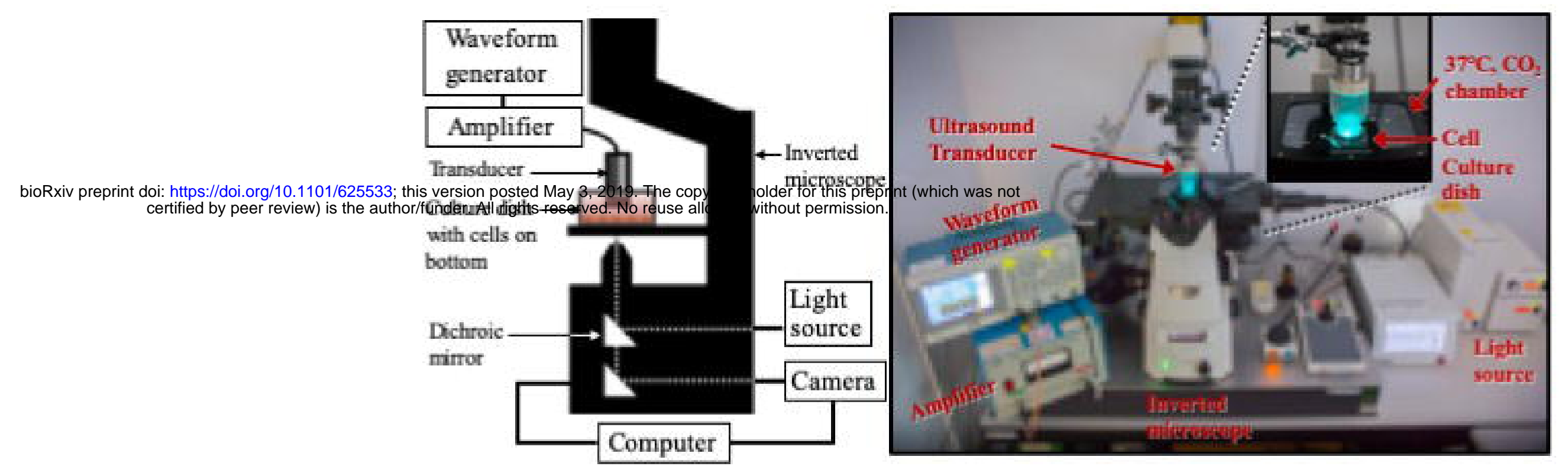




\section{Extended Figure 2}

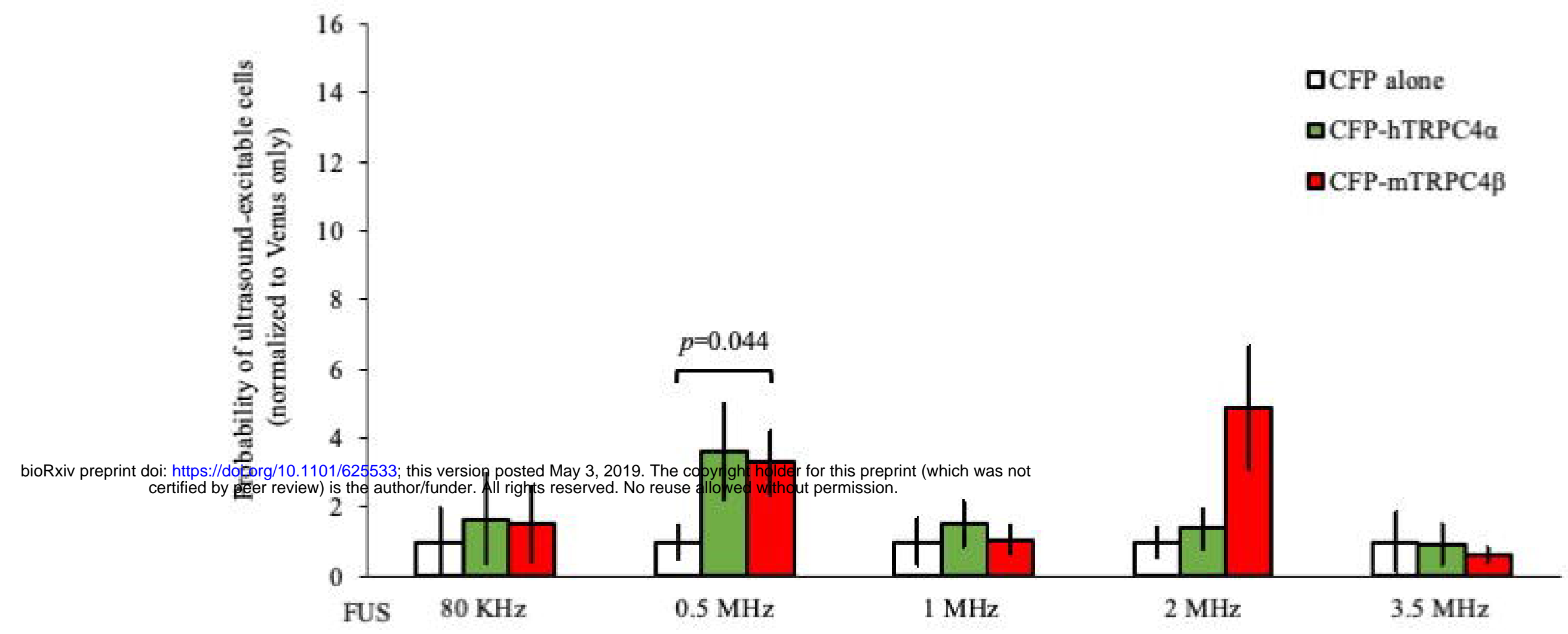




\section{Extended Figure 3}

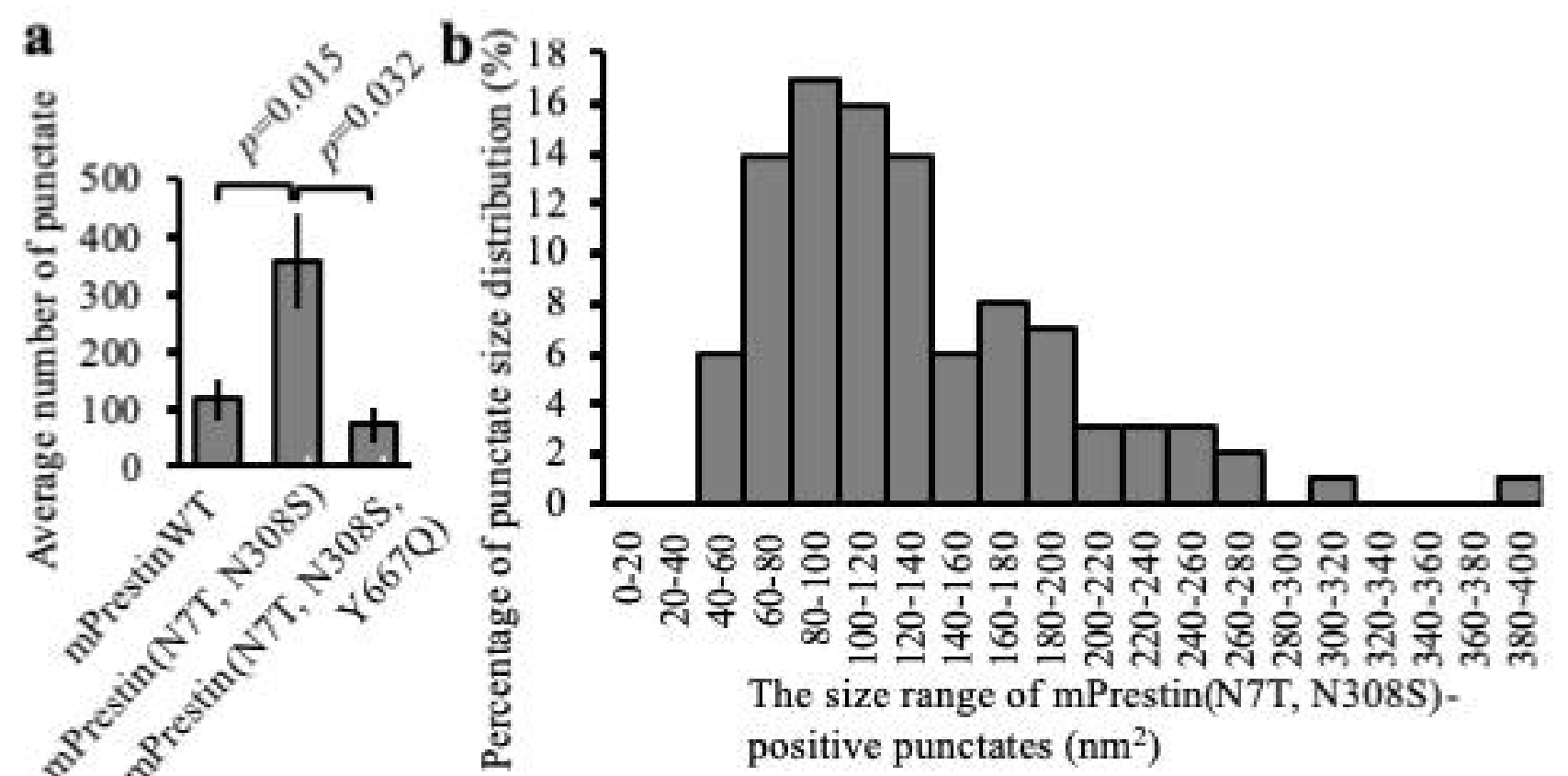

C Venus-mPrestinWT Venus-mPrestin(N7T, N308S) d

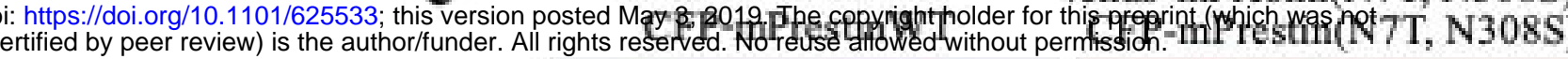

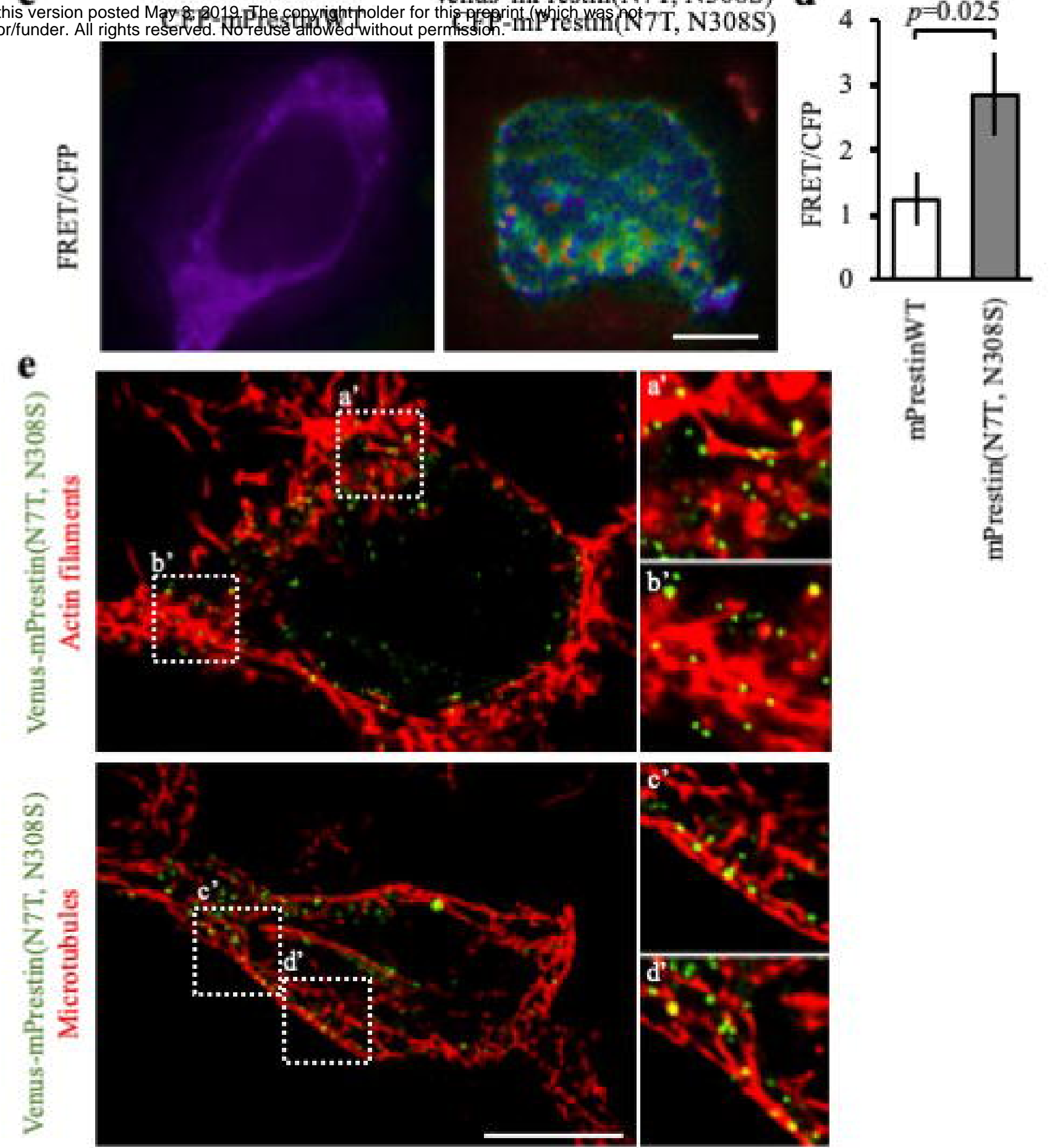




\section{Extended Figure 4}

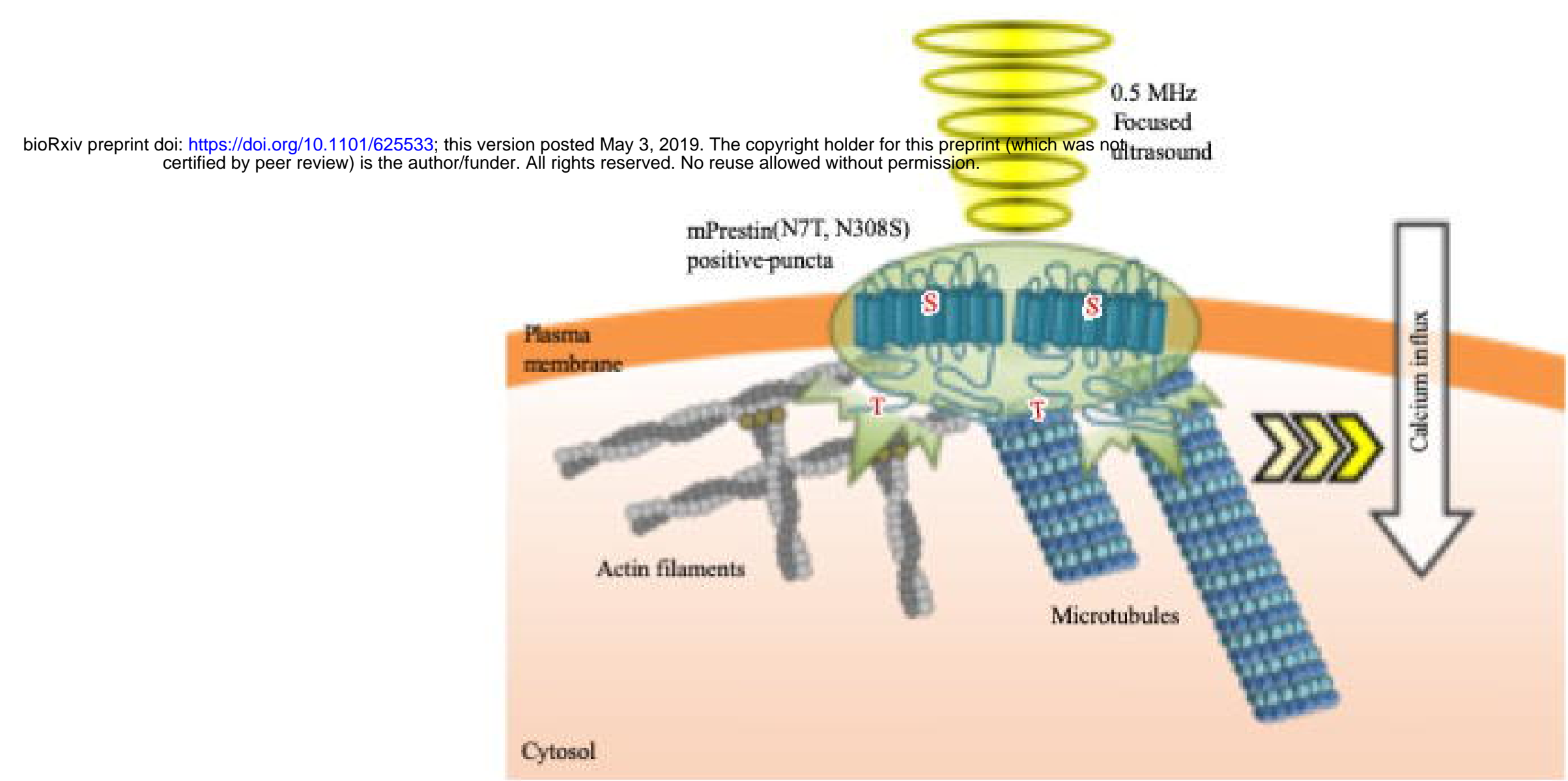

